\title{
Das Ende des Neuen Reiches ${ }^{1}$
}

\section{Herihor und Pianch}

Als die Schlüsselfigur beim Übergang von der 20. zur 21. Dynastie ist von jeher der Hohepriester Herihor angesehen worden, obwohl nur sehr wenig von ihm bekannt ist, in zweiter Linie dann auch sein angeblicher Sohn und Nachfolger Pianch. Der Grund dafür ist einfach: Im Chonstempel von Karnak erscheint Herihor zunächst als Hoherpriester und Bauleiter für Ramses XI., dann aber selbst als König. Die frühere Annahme war natürlich, er habe nach dem Tod des Königs den Thron usurpiert (und ihn dann mit Smendes im Norden geteilt) ${ }^{2}$. Herihor ist auch wie selbstverständlich als spiritus rector der „Erneuerungsära" wḥm mswt angesehen worden ${ }^{3}$, nach der im letzten Abschnitt der Regierung Ramses’ XI. überwiegend datiert wird.

$\mathrm{Da}$ der Hohepriester und General Pianch, der in dieser Zeit ebenfalls eine große Rolle spielt, der Sohn des Herihor sei, ergab sich anscheinend gleichfalls aus der Dekoration des Chonstempels: In einer Darstellung einer großen Zahl von Kindern des Herihor, der „Prinzenprozession“, hatte Lepsius' Abschrift an prominenter Stelle eine Namensbeischrift [ ]- $n h$ busgemacht ${ }^{4}$, die natürlich $\left[P_{3 j} j\right]^{\text {' }} n b$ gelesen wurde, später in Gauthiers Königsbuch ${ }^{5}$ sogar ganz ohne Angabe einer Ergänzung. Der Beginn der Dominanz des Herihor ist nach allgemeiner Ansicht frühestens ab Jahr 17 Ramses' XI. möglich, da aus diesem Jahr noch ein Brief Ramses XI. an den Vizekönig Panehsi datiert, der damals noch der Herr der Thebais gewesen sein $m u \beta^{6}$. An festen Daten sind für Herihor nur Jahr 5 (Wenamun, 1,1) und Jahr 6 („Dockets“ auf den Särgen Sethos’ I. und Ramses’ II. ${ }^{\text {) }}$ überliefert, ohne ausdrücklichen Bezug auf einen König oder eine Ära, aber allgemein der whm mswt zugerechnet.

Nun ist 1948 eine Inschrift veröffentlicht worden ${ }^{8}$, die nicht ganz zur bisherigen Theorie paßte: Sie stammt aus dem Jahr 7 der whm mswt (= Jahr 25 Ramses XI.) und zeigt den Pianch als Hohenpriester des Amun. Also, so die Schlußfolgerung, ist Herihor spätestens im Jahr 7 gestorben und hat Pianch Platz gemacht ${ }^{9}$. Er müßte sich dann schon zu Lebzeiten Ramses XI. als König haben darstellen lassen.

Pianch seinerseits ist chronologisch durch dieses Datum fixiert, er ist weiterhin (indirekt) im Jahr 10 (sicher der wḥm mswt) in den Late Ramesside Letters bezeugt (Brief Nr. 9, s. a. Wente, LRL, p. 12). Bei der Neupublikation des Chonstempels durch den Chicagoer Epigraphic Survey hat sich nun gezeigt, daß in

\footnotetext{
${ }^{1}$ Abkürzungen nach Lexikon der Ägyptologie, Bd. VII, p. XIV ff. Außerdem TIP = K. A. Kitchen, The Third Intermediate Period in Egypt, ${ }^{2}$ Warminster 1986; Wente, LRL=E. F. Wente, Late Ramesside Letters, SAOC 33, 1967; ToK= The Temple of Khonsu. By the Epigraphic Survey, Bd. I/II, OIP 100/103, Chicago 1978/1981; KRI = K. A. Kitchen, Ramesside Inscriptions, Oxford 1968ff.; Niwiński, Coffins=A. Niwiński, $21^{\text {st }}$ Dynasty Coffins from Thebes, Theben 5, 1988.

${ }^{2}$ Z. B. E. Meyer, SPAW 1928, p. 495-7.

${ }^{3}$ Vgl. v. a. Kees, Herihor und die Aufrichtung des thebanischen Gottesstaates, NGWG 1936, 1-20.

${ }^{4}$ LD III, 247a.

${ }^{5}$ LR, III, 237, XIX, A.

${ }^{6}$ S. z. B. TIP $\int 208-9$.

7 S. TIP $\int 379,2-3$.

${ }^{8}$ JNES 7, 157-62.

9 Kees, Hohenpriester, p. 14-5, hat dagegen angenommen, Pianch sei zu dieser Zeit noch nicht Hoherpriester gewesen - was klar dem Wortlaut der Beischrift widerspricht. Nims, der Erstbearbeiter des Textes, hat vermutet, die Inschrift könne eine Weile nach dem Ereignis angebracht worden, also anachronistisch sein, und Pianch sei erst in der Zwischenzeit Hoherpriester geworden. Die heute allgemein vertretene Ansicht ist aber wohl die von Kitc hen, TIP \$16-8, daß Herihor tatsächlich schon im Jahr 7 gestorben ist und dem Pianch Platz gemacht hat. Neuerlich will Bell, Serapis 6, 1980, 18ff. (bes. 24) wieder eine gewisse - wenn auch nur kurze - Zeit zwischen Ereignis und Gravierung der Inschrift annehmen. Seine Argumente (zum Nesamun-Problem insgesamt) scheinen mir aber etwas weit hergeholt.
} 
der Prinzenprozession Pianch gar nicht vorkommen kann: An der fraglichen Stelle ist vor ${ }^{\mathrm{n}} \mathrm{h}$ k keine Lücke, der Name ist entweder ${ }^{C} n b[. f]$ oder ${ }^{~} n b[. f-n-M w t]$ zu lesen ${ }^{10}$, ein auch sonst bezeugter Sohn des Herihor ${ }^{11}$.

Wenn man das Material einmal unvoreingenommen und unbeeinflußt von dem folgenreichen Kopierfehler der Lepsius-Expedition und den darauf aufbauenden Schlußfolgerungen betrachtet, ergibt sich bezüglich des Verhältnisses von Herihor und Pianch ein ganz anderes Bild, und es wird deutlich, daß Pianch zeitlich vor Herihor anzusetzen ist.

1. Es ist weitgehend unstrittig, daß die reale Macht der Hohenpriester der 21. Dynastie und ihrer Vorgänger Herihor und Pianch am Ende der 20. Dynastie auf ihrer militärischen Macht, also ihrem Generalsrang, basierte und daß ihre Träger ursprünglich aus dem Offiziersstand kamen. Kees ${ }^{12}$ nennt ihre Herrschaft sogar eine „als Wille des Amun getarnte Militärdiktatur“. Was speziell Pianch und Herihor angeht, so ist folgendes bemerkenswert ${ }^{13}$ : Pianch ist offenbar ein reiner Militär. Sein Generalsrang steht absolut im Vordergrund: wird nur ein Titel genannt, heißt er schlicht „der General“ oder „der General des Pharao"14. Der Titel des Hohenpriesters dagegen erscheint zu seinen Lebzeiten relativ selten (ganz anders dagegen in den postumen Erwähnungen seines Sohnes Pajnedjem!). In den Late Ramesside Letters und den thebanischen Graffiti wird oft nur „der General“ erwähnt, und es ist klar, daß damit nur Pianch gemeint sein kann. Selbst in der erwähnten Orakelinschrift aus dem Jahr 7 wird seine vollständige Titulatur nur in der Beischrift gegeben; im eigentlichen Text ist er wieder einfach „der General“15.

Ganz anders bei Herihor: Bei ihm steht der Hohepriestertitel ganz deutlich im Vordergrund, er wird immer mit ihm verbunden, der Generalstitel dagegen nur bei vollständigerer Anführung der Titulatur. Außerdem sind Pianchs militärische Titel detaillierter: Neben den Titeln $m r m \check{s}^{\breve{c}}$ und ḩswtj (beide auch bei Herihor) führt er noch den besonderen Rang eines bswtj $n$ pd dwt $p r-{ }^{9} 3^{16}$ bzw. hrj-pdwt $p r-{ }^{-} 3^{17}$.

2. Pianchs $\operatorname{Titel}^{18}$ (v. a. seine wichtigen militärischen Titel!) sind sehr oft auf den regierenden König (also Ramses XI.) bezogen: Er heißt $m r m \breve{s} n$ pr-3 (neben bloßem $m r m \breve{s}$ ), b̧swtj $n$ pd $w t p r-3$, brj-pdudwt $p r-3, m r \check{s} n w t j n$ pr- ${ }^{-}$, Herihor ${ }^{19}$ dagegen immer nur $m r m \breve{s}$ (wr) und $m r \check{s} n w t j$ oh ne $p r-{ }^{e} 3^{20}$. Gemeinsam sind beiden allerdings die königlichen Titel țjj-b̧w ḥr wnm njswt und $\chi^{5}$ njswt, doch hier gehört das njswt fest zum Titel. Ferner führt Herihor Beiworte wie $m h-j b$ ' $3 n n b t 3 w j \mid n \underline{t} n n f r$, aber das besagt nicht viel. Die wirklich entscheidenden militärischen und administrativen Titel sind bei Pianch noch deutlich auf den regierenden König bezogen, bei Herihor nicht mehr!

3. Sowohl Herihor als auch Pajnedjem I., der Sohn des Pianch, nehmen königliche Titel und Attribute

${ }^{10}$ S. ToK, I, pl. 26, Z. 4; Übersetzungsheft, p. 13 (d); Wente, Drevny Vostok (Fs Korostovtsev), Moskau 1975, p. 36-8.

${ }^{11}$ ToK, I, pl. 44, 10-12. Es ist nicht sicher, ob die einmalige Bezeichnung des Pianch als $₹ 3$ njswt $n$ bt.f auf einer Statue in Privatbesitz (BSEG 7, 1982, 38-46) ernstzunehmen ist, da es sich bei dieser Statue (bzw. der Inschrift) um eine Fälschung handeln könnte, vgl. Dewachter, BSEG 11, 1987, 3-5.

12 Op. cit., p. 2.

${ }^{13}$ Eine ausführliche Untersuchung der Titulaturen der Hohenpriester der 21. Dynastie (inklusive Pianch und Herihor) in der (noch unpublizierten) Berliner Habilitationsschrift von M. Römer, Studien zur Gottes- und Priesterherrschaft in Ägypten, $\int 34-63$.

${ }^{14}$ Für die Late Ramesside Letters z. B. s. BIFAO 78, 1978, 207-12.

${ }^{15}$ Die komplizierten Folgerungen, die Bell, op. cit., p. 18ff., und andere daraus gezogen haben, sind unnötig.

${ }^{16}$ LRL 44,$5 ; 61,16 f$.

${ }^{17}$ KRI VI, 848, 16; BSEG 7, 1982, 45 [?].

${ }^{18} \mathrm{Zu}$ den Titeln des Pianch s. v. a. KRI VI, 848, 15-16 (Stele aus Abydos); 702, 8/13 (Orakeltext Karnak); 849, 2-4 (Ostrakon CG 25745); LRL, 44,3-5 (Brief Nr. 28); BSEG 7, 1982, 45 (Statue in Privatbesitz [echt??]) sowie allgemein die Zusammenstellung in BIFAO 78, 1978, 197-212.

${ }_{19} \mathrm{Zu}$ den Titeln des Herihor s. v. a. KRI VI, 847, 10-3 (Ostrakon CG 25744); 709, 8-9; 710,3 (Orakeltext Chonstempel); ToK pl. 153; 163; 166; 172; 174; 178A; 185; 188; 195-6; 198-199; 201; KRI VI, 843,15-844,2 (Statue CG 42190); 846,5 (Graffito Karnak); 847,8 (Sphinx Karnak); 846,8-847,6 (Stele Leiden V 65); Karnak V, p. 10, fig. 5; Wenamun, 2,61; Maspero, Momies Royales, p. 553; 557 („Dockets“ auf den Särgen von Sethos I. und Ramses II.). Herihor ohne Titel: Wenamun 1,15; 2,25-6. Zusammenstellung bei Lefebvre, Grands Prêtres, p. $272 \mathrm{ff}$.

${ }^{20}$ Kitchen, KRI VI, 847, 12 ergänzt einmal $n$ pr-'3, aber das ist dem verbliebenen Raum nach unwahrscheinlich, s. Černý, Ostraca hiératiques (CG), pl. XCII. 
an, später auch noch der Hohepriester Mencheperre. Pianchi tut dies hingegen nie, was immerhin erstaunlich wäre, läge er zeitlich zwischen Herihor und Pajnedjem.

4. Von Herihor bis Pajnedjem II. sind alle Hohenpriester (Herihor, Pajnedjem I., Masaharta, Mencheperre, Smendes II, Pajnedjem II., Psusennes „III.“) auf Särgen und Leichentüchern (etc.) der wiederund neubestatteten Mumien in den Massengräbern von Theben-West bezeugt, mit der einzigen Ausnahme von Pianch ${ }^{21}$ ! Die Bestattungen setzen offenkundig erst nach seiner Amtszeit ein.

5. Sowohl Herihor als auch Pajnedjem I. sind in Theben als Bauherren und Restauratoren bezeugt ${ }^{22}$, Pianch dagegen nicht.

Das ist besonders auffällig für den Bereich des Chonstempels von Karnak, wo man einen offenbar bruchlosen Übergang: Herihor als Hoherpriester im Namen Ramses XI. - Herihor als König - der Hohepriester Pajnedjem hat. Auch hier kommt Pianch überhaupt nicht vor.

Alle Indizien sprechen soweit dafür, daß Pianch der Vorgänger des Herihor ist, nicht sein Nachfolger. Weitere Überlegungen können diesen Schluß nur stützen:

6. In den aus der wḥm-mswt-Zeit stammenden Grabräuberpapyri (pBM 10383; 10052; 10403; Abbot Dokkets; Mayer A; Ambras) wird zwar die Institution des Hohenpriesters verschiedentlich erwähnt, aber kein Amtsinhaber. Während diese Tatsache verständlich wäre, wenn Pianch Hoherpriester war (denn er führte um diese Zeit wohl Krieg in Nubien), wäre es doch sehr verwunderlich für den Fall, daß Herihor damals Hoherpriester und der „starke Mann“ war. Übrigens wird Herihor an keiner Stelle irgendwie mit der whm-mswt-Ära in Zusammenhang gebracht.

7. Unmittelbarer Vorgänger von Pianch und Herihor als Machthaber der Thebais war bekanntlich der Vizekönig Panehsi, der in der $w h m-m s w t$-Zeit in Ungnade gefallen ist und von Pianch in Nubien bekämpft wird. Ein Vergleich seiner Titulatur mit der des Pianch und des Herihor (sowie der späteren Hohenpriester) ist äußerst aufschlußreich, denn es zeigt sich eine deutliche Entwicklung von Panehsi über Pianch und Herihor zu den späteren Hohenpriestern.

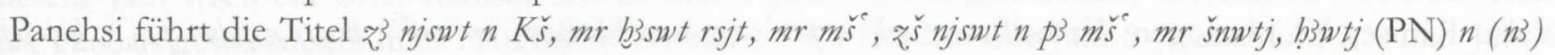

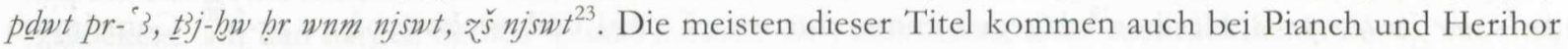
$\operatorname{vor}^{24}$, mit Ausnahme von $z^{s}$ njswt $n$ p 3 m $\check{s}^{\circ}$. Den Titel des Obersten der Bogenschützen gibt es allerdings nur bei Pianch, und zwar genau in der Form wie bei Panehsi als ḩ3wtj (PN) $n 3 p d w t p r-3^{25}$ und außerdem noch in dem wohl gleichbedeutenden brj pdw Hohenpriestern nicht). Weiter heißen sowohl Panehsi als auch Pianch nur $m r m \breve{s}$ bzw. (Pianch) $m r m \breve{s}$

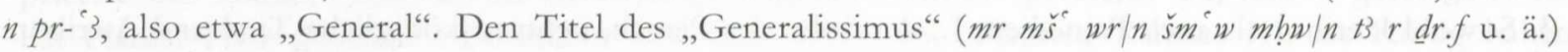
gibt es erst bei Herihor und dann bei allen folgenden Hohenpriestern, und zwar immer ohne Bezug auf den König wie noch bei Pianch ${ }^{27}$. Die Titulatur des Panehsi ist also mit der des Pianch deutlich näher verwandt als mit der des Herihor.

Die Titel, worin Pianch und Herihor sich v. a. von Panehsi unterscheiden, sind 1. der Hohepriestertitel und 2. der Wezirstitel (den nach diesen beiden von den Hohenpriestern dann nur noch Pajnedjem führt). Übrigens werden nach Herihor auch viele der Titel, die beim Übergang von Dynastie 20 zu 21 offenbar von Panehsi übernommen worden waren (s. u.), von den folgenden Hohenpriestern nicht mehr geführt,

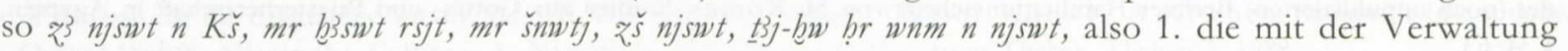
Nubiens verbundenen Titel, 2. die königlichen Ehrenämter und 3. der besondere Fall des „Speichervorstehers" (s. dazu unten).

$\mathrm{Zu}$ dieser engeren Verwandtschaft der Titulaturen des Panehsi und des Pianch (gegenüber Herihor)

${ }^{21}$ S. die „datelines“ in TIP $\int 379 \mathrm{ff}$.

${ }^{22}$ Zu Herihor s. LÄ Iқ, 1132 oben und Karnak V, 10; zu Pajnedjem I. s. TIP \& 215-6.

${ }^{23}$ S. RAD, 36, 4-5; KRI VI, 734, 10. In einem Graffito in Buhen (KRI VI, 842, 10-12) zusätzlich noch die Titel jrj-p’t bistjund $m r$ pr Jmn, falls es überhaupt zu ihm gehört, was Caminos, The New-Kingdom Temples of Buhen, II, 109-10 ablehnt.

${ }^{24}$ Die Nachweise ihrer Titel oben in Anm. 18 und 19.

${ }^{25}$ LRL 44,5; 61,16; BSEG 7, 1982, 45[?].

${ }^{26}$ KRI VI, 848, 16.

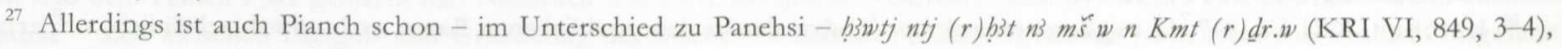
ebenso Herihor und (später) der Hohepriester Mencheperre. 
kommt noch, daß die Konfrontation Panehsi-Pianch ja belegbar ist ${ }^{28}$, irgendeine Beziehung zwischen Panehsi und Herihor dagegen nicht.

8. Ein weiterer Punkt, der für eine Folge Pianch - Herihor spricht, sind die genealogischen Informationen. Die Eltern beider sind unbekannt, aber die Frau und spätere Königin des Herihor war die bekannte Nedjemet $^{29}$, die als Königin und Königsmutter bezeichnet wird. Die Mutter dieser Nedjemet wiederum ist eine Königsmutter Hrrt. Was die Frau des Pianch betrifft, so ist sicher, daß ihr Name mit einem $b$ beginnt ${ }^{30}$. In Brief Nr. 2 der Late Ramesside Letters wird Pianch von einer Dame namens Hrrt vor einem Feldzug nach Elephantine begleitet ${ }^{31}$. Der alten Vorstellung folgend, Pianch sei Herihors Sohn, haben die Bearbeiter des Briefes angenommen, diese Hrrt sei die Mutter der Nedjemet, also Pianchs Großmutter. Bierbrier ${ }^{32}$ hat zurecht darauf hingewiesen, wie unwahrscheinlich es ist, daß ein General seine doch sicher betagte Großmutter mit auf eine Feldzugsvorbereitung nimmt. Er hat deshalb eine Hrrt „II“ als Frau des Pianch angesetzt ${ }^{33}$. Das ist jetzt überflüssig: Es ist nur noch eine Hrrt nötig, eben die Frau des Pianch und somit Schwiegermutter des Herihor (und „Königsmutter“ des Pajnedjem). Anders gesagt, Herihor ist der Schwiegersohn des Pianch. Mithin stimmen auch die verfügbaren genealogischen Daten bestens zur Folge Pianch - Herihor. Man vergleiche auch Niwiński, JARCE 16, 1979, 52 (mit n. 21 [p. 62]), der das genealogische Verhältnis der Frauen von Herihor und Pianch ebenso ansetzt.

Es kann m. E. nicht den geringsten Zweifel daran geben, daß Herihor der Nachfolger (und Schwiegersohn), nicht der Vorgänger des Pianch ist.

Chronologisch hieße das, daß Pianch nach dem Jahr 17 Ramses' XI. den Vizekönig Panehsi verdrängt (und in seinen Machtbefugnissen „beerbt") hatte, und dann bis zum Jahr 10 (der $w h h^{c} m s w t=\mathrm{Jahr} 28$ Ramses' XI.) belegt ist. Spätestens im Jahr 7 whm mswt, wahrscheinlich aber schon vorher, ist er auch Hoherpriester des Amun.

Wohl nicht allzulange nach dem Jahr 10 wird Herihor seinem Schwiegervater in diesem Amt gefolgt sein, sicher noch zu Lebzeiten Ramses’ XI., wie sich aus der Dekoration des Chonstempels ergibt. Erst nach dessen Tod dürfte Herihor sich den Königstitel zugelegt haben. Im Zusammenhang mit Herihor sind nur wenige Daten überliefert: Jahr 5 (Wenamun) und Jahr 6 (Sargdockets) ${ }^{34}$, die bisher in die $w h m-m s w t-A ̈ r a ~ g e s e t z t$ worden sind. Diese Datierung hat schon immer eine gewisse Schwierigkeit gemacht: Wenn die Erzählung und das Datum des Wenamun auf historischen Fakten beruhen, wie allgemein angenommen wird und auch wohl zutreffen dürfte ${ }^{35}$, hatte Herihor im Jahr 5 eine Gesandtschaft in den Libanon geschickt, um Holz für die Barke des Amun zu holen. In der Dekoration des Chonstempels ${ }^{36}$ wird diese Barke abgebildet und gesagt, daß Herihor sie aus Holz des Libanon anfertigen ließ. Der „Wenamun“ und diese Angabe bestätigen sich also gegenseitig. Wenn Herihor aber im Jahre 7 schon tot war, wie bisher angenommen, hätte er die Konstruktion der Barke kaum noch erleben können und erst recht nicht die Bezugnahme darauf in der Tempeldekoration. Um dieser Schwierigkeit zu begegnen, hat Kitchen angenommen ${ }^{37}$, daß die Darstellung ,with lively anticipation“ angebracht wurde, also u. U., bevor das Holz überhaupt Theben erreicht hatte. Auch Wente ${ }^{38}$ hat sich dem angeschlossen.

Wie dem auch sei, der Ansatz der Daten in die whbm-mswt-Ära ist nach den obigen Ausführungen, mit

${ }^{28}$ S. LRL, 7,16-8,1; Wente, LRL, p. 25, n.g.

29 S. TIP \& $37-9$.

${ }^{30}$ S. Rec'Trav 32, 1910, 185.

${ }^{31}$ LRL $3,7-8$.

32 JNES 32, 1973, 311.

33 Ebenso TIP § 39 und 438: „Hrere B“.

${ }^{34}$ S. TIP $\int 379$.

${ }^{35}$ Obwohl es sich ohne Zweifel um einen literarischen Text, nicht um einen Originalbericht handelt, s. zuletzt Osing, Nubia et Oriens Christianus (Fs C. D. G. Müller), Köln 1988, p. 37-9.

${ }^{36}$ ToK. pl. 21; 143, C2.

37 TIP S 210.

${ }^{38}$ Übersetzungsheft zu ToK I, p. XIV. 
Pianch als Vorgänger des Herihor, auf keinen Fall mehr aufrechtzuerhalten. Es bleiben dann nur zwei Möglichkeiten:

1. Es handelt sich um die Jahre 5 und 6 des Smendes.

Dagegen könnte man einwenden, wie es Kitchen getan hat ${ }^{39}$, daß im Wenamun der Name des Smendes ohne Kartusche geschrieben wird und er auch sonst in keiner Weise als König bezeichnet wird. Dieser Einwand wiegt allerdings angesichts des satirischen Charakters dieses Textes ${ }^{40}$ nicht schwer; eine korrekte und feierliche Bezeichnung ist hier eigentlich gar nicht zu erwarten ${ }^{41}$. Auch Herihor wird ja zweimal $(1,15 ; 2,15-6)$ nur mit seinem Namen erwähnt: In offiziellen Inschriften wäre das etwas ganz Unmögliches. Ebenso wird in 2,51; 53 ein $H^{e} j-m$-W3st erwähnt, womit wohl am ehesten Ramses IX. oder Ramses XI. gemeint sein dürfte (kaum der gleichnamige Wezir). Auch hier keinerlei Titel oder Andeutung, daß es sich um einen König handelt.

Der zweite Einwand könnte sich auf die „datelines“ stützen: Der Hohepriester Pajnedjem ist in einem Jahr 6, 3.prt, 7 belegt $^{42}$, das nach Kitchens System nur Smendes zugerechnet werden kann. Demnach dürfte Herihor nicht im Jahr 6, 3.prt, $15^{43}$ ebenfalls unter Smendes belegt sein. Es ist aber nicht sicher, ob hier wirklich prt zu lesen ist: Daressy ${ }^{44}$ liest nämlich $3 h t^{45}$. Geht man von einer Lesung $3 b t$ aus, wäre es möglich, die Daten des Herihor auf Smendes zu beziehen. Als Konsequenz daraus wäre dann der Wechsel von Herihor zu Pajnedjem ziemlich genau zu datieren (zwischen 3.3bt, 15 und 3.prt, 7 im Jahr 6 des Smendes) $)^{46}$. Das oben angesprochene Problem der indirekten Erwähnung der Wenamun-Expedition bliebe bestehen, man müßte dann Kitchens Lösungsvorschlag akzeptieren.

Es wäre also möglich, Kitchens Datierungssystem für die 21. Dynastie im Grundsätzlichen aufrechtzuerhalten, auch wenn Herihor auf Pianch folgt ${ }^{47}$.

2. Dennoch scheint mir diese Konstruktion im ganzen eher unwahrscheinlich. Ich werden unten (Teil III) zu zeigen versuchen, daß eine zweite Möglichkeit die wahrscheinlichere ist: Die Datierungen des Herihor geben seine eigenen Regierungsjahre an.

\section{Die Ereignisse am Ende der 20. Dynastie}

Es ist oben schon kurz angesprochen worden, daß Pianch in gewisser Weise den Panehsi als Machthaber in der Thebais „beerbt" hat. Fest steht, daß Panehsi spätestens zu Beginn der whm-mswt-Ära verfemt war ${ }^{48}$ und von Pianch in Nubien bekämpft wird ${ }^{49}$.

Es fragt sich nun, wie es zu dieser Verfemung gekommen ist, in deren Gefolge Pianch und sein Nachfolger Herihor eine solche Machtfülle sammeln konnten, daß Herihor sich schließlich selbst als König darstellen ließ. Die Texte enthalten folgende Hinweise:

1. Im „Turin Taxation Papyrus“ aus dem Jahr 12 Ramses’ XI. ist Panehsi mit den für seinen Posten äußerst ungewöhnlichen Titeln „Speichervorsteher" und „General“ in Theben anwesend und trifft offenbar Verfügungen im Zusammenhang mit Getreideabgaben ${ }^{50}$.

2. Im Jahr 17 schreibt Ramses XI. einen Brief an ihn mit der Aufforderung, einen königlichen Beauftragten

39 TIP $\int 14$.

${ }^{40}$ S. Osing, op. cit., p. 39.

${ }^{41}$ Um so mehr, als sich die Ägypter des merkwürdig unvollkommenen Königtums dieser Dynastie, zumal an ihrem Beginn, sicher bewußt gewesen sind.

${ }^{42}$ S. TIP § $381,9$.

43 TIP $\int 379,3$.

${ }^{44}$ Cercueils des cachettes royales (CG), p. 32.

${ }^{45}$ Das Photo, ibid., pl. XXII, erlaubt leider keine Überprüfung.

${ }^{46}$ In TIP $\$ 379,2$ ist 2. prt in 2. 3bt zu korrigieren, s. Maspero, Momies Royales, p. 553 und Daressy, op. cit., p. 30 und pl. XVIII.

47 Das ominöse Band mit „a reference to the first year of Pinotmou“ (Smith, The Royal Mummies (CG), Kairo 1912, p. $\left.97=\operatorname{TIP} \int 381,6\right)$ müßte dann nicht Smendes, sondern Psusennes zuzuordnen sein, wie es (aus anderen Gründen) Niwiński, JARCE 16, 1979, 52-3 (= Coffins, p. 43) vorgeschlagen hat.

${ }^{48}$ Vgl. pBM 10052, 10,18; 10383, II,5; pMayer A, 13,B3.

${ }^{49}$ LRL 7,16-8,1.

${ }^{50}$ RAD, p. 36. 
beim Transport eines Kultgegenstandes zu unterstützen ${ }^{51}$. Der Brief ist, wie Gardiner bemerkt hat, in ziemlich barschem Ton gehalten ${ }^{52}$. Im Jahr 17 ist Panehsi dennoch offenbar persona grata, im Jahr 19 nicht mehr.

3. In den Grabräuberpapyri gibt es einige Andeutungen über einen Krieg und über einen Angriff (o. ä.) auf den Hohenpriester Amenhotep.

- pMayer A, 6, 4-8 ${ }^{53}$ : (Ein Zeuge sagt:) „Die Barbaren kamen und eroberten den Tempel (= Medinet Habu), als ich Esel meines Vaters hütete, und Pahati, ein Barbar, ergriff mich und verschleppte mich nach Jpjp, als Amenhotep, der Hoherpriester war, (schon) sechs Monate lang attackiert worden war. Und es geschah, daß ich (erst nach) neun Monaten des Angriffs gegen Amenhotep, der Hoherpriester war, zurückkehrte (als dieser tragbare Schrein [schon] beschädigt war und man Feuer an ihn gelegt hatte ....).“

- pMayer A, 13, B, 2-3:

„Diebe, die im Krieg im Norden ( $m$ htj) getötet worden sind: 15 Mann;

Diebe, die Panehsi getötet hat: 3 Mann.“

- pMayer A, 4, 4-5:

(Ein Zeuge sagt:) „Ich floh vor den $m d w-{ }^{-} n$ [+ Personendeterminativ und Pluralstrichen], als Panehsi die $m d w$ - $n$ machte."

- pBM 10052, 13, 24:

„... als man den Krieg des Hohenpriesters/gegen den Hohenpriester machte ...“

- pBM 10052, 10, 18-20:

(Ein Sklave sagt als Zeuge:) „Als Panehsi Kynopolis zerstörte, da kaufte mich der junge Nubier Bwthj-Jmn, und der Nubier P3-n-t3-sbg kaufte mich von ihm, und er bezahlte $2 d b n$ Silber für mich. Und als man ihn tötete, da kaufte mich der Gärtner $K r$.“

- pBM 10383, II, 5:

(Ein Wächter des Hauses des Pharao sagt:) „Ich verließ das Haus des Pharao, als Panehsi kam und meinen Vorgesetzten angriff, obwohl er kein Vergehen begangen hatte.“

Man vergleiche auch:

- pBM 10052, 11, 7-8:

(Eine Zeugin sagt:) „Ich kaufte dafür Getreide in dem Jahr der Hyänen, als man hungerte.“

Es ist also die Rede 1. von inneren Unruhen, in deren Verlauf Medinet Habu erstürmt und der Hohepriester Amenhotep 9 Monate lang „bedrängt“ wurde, und 2. von einem Krieg im Norden, d. h. in Unterägypten, bzw. bis nach Unterägypten ( $m b t^{54}$ ), in dessen Verlauf u. a. Kynopolis zerstört wurde. Dieser Krieg im Norden wird von Panehsi geführt. In welcher Beziehung der Krieg zu dem Angriff auf den Hohenpriester steht und ob Panehsi auch dabei eine Rolle spielt, geht aus den Äußerungen nicht klar hervor. Allerdings läßt pBM 10383, II, 5 doch darauf schließen, daß auch bei diesem Vorgang Panehsi der Übeltäter war (sofern mit $p 3 j . j$ ḅrj hier der Hohepriester gemeint ist, was an sich naheliegt).

4. 1966 hat Wente einen sehr fragmentarischen Text aus Karnak wiederveröffentlicht ${ }^{55}$, in dem ein Hoherpriester, bei dem es sich nur um Ramsesnacht oder Amenhotep handeln kann (da der Totentempel Ramses VI. erwähnt wird), schildert, wie er von einem Feind, dessen Name und Bezeichnung leider nicht erhalten sind, 8 Monate lang bedrängt wird. Wente hat klar gezeigt, daß es sich bei dem Hohenpriester nur um Amenhotep handeln $\mathrm{kann}^{56}$ : Es wäre ein zu großer Zufall, wenn Ramsesnacht 8 Monate und sein Nachfolger 9 Monate „unterdrückt“ worden wären. Wenn es sich um denselben Vorfall handelt, erklärt sich die Diskrepanz leicht: Im einen Fall hat man aufgerundet, im anderen abgerundet. Besonders

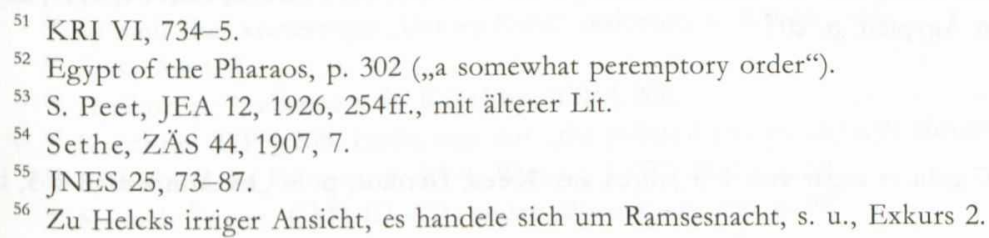


wichtig ist nun, daß Amenhotep sagt, er habe sich an den König um Hilfe gewandt (Z. 21), und daß der Angriff schließlich erfolgreich abgewehrt worden ist (Z. 24).

Die naheliegendste und einfachste Art, die Fakten von Nr. 1-4 zusammenzubringen, wäre nun die folgende:

Derjenige, der den Hohenpriester Amenhotep attackiert und „unterdrückt“, ist Panehsi, und der „Krieg des Hohenpriesters" ist eben diese Auseinandersetzung zwischen ihnen, bei der auch Medinet Habu erstürmt und z. T. zerstört wurde. Der Hohepriester wendet sich daraufhin um Hilfe an den König, und im Verlauf dieser Auseinandersetzung kommt es zur Zerstörung von Kynopolis und zum Krieg im Norden, und schließlich endet der Krieg spätestens im Jahre 19 mit der Vertreibung des Panehsi aus Ägypten durch den königlichen General Pianch, der daraufhin alle Ämter des Panehsi an sich zieht. Auf diese Weise wäre klar, warum Panehsi im Jahr 19 in Theben verfemt war.

Eben diese einfache Erklärung ist aber bisher von keinem Historiker in Betracht gezogen worden; alle setzen die Unruhen um den Hohenpriester (z. T. wesentlich) früher als im Jahr 17 an, und zwar mit den unterschiedlichsten Argumenten.

- Zunächst einmal sind inzwischen alle Theorien hinfällig, die davon ausgingen, Panehsi habe vor dem Jahr 12 (im Auftrag des Königs) den zu mächtig gewordenen Hohenpriester ${ }^{57}$ zur Raison bringen sollen, so z. B. von Helck ${ }^{58}$ und Černy ${ }^{59}$. Der von Wente publizierte Text aus Karnak zeigt eindeutig, daß der Hohepriester sich an den König um Hilfe wendet und sein Opponent daraufhin erfolgreich abgewehrt wird (während Panehsi ja bis zum Jahr 17 unangefochten ist). Cerný will die ungewöhnliche Tatsache, daß der Vizekönig Panehsi (zumindest) ab Jahr 12 in Theben ist und - wie oben erwähnt - unübliche Titel führt, dadurch erklären, daß er damals bereits gegen den Hohenpriester eingeschritten war. Dieser Sachverhalt läßt sich jedoch auch anders deuten (s. u.).

- Aldred ${ }^{60}$ setzt den „Krieg des Hohenpriesters“ zwischen Jahr 8 und 9 Ramses’ XI. an, denn im Jahr 8 wird die Zahl der Nekropolenarbeiter deutlich reduziert ${ }^{61}$. Das erklärt er damit, daß sie (von seiten des unbekannten Aggressors) zur Armee eingezogen wurden, um gegen den dem Hohenpriester zu Hilfe eilenden Panehsi zu kämpfen. Die Tatsache der Reduktion läßt sich jedoch viel einfacher mit Versorgungsmängeln erklären (s. u.).

- Peet hat versucht, aus der internen Evidenz des pMayer A, wo die Eroberung Medinet Habus und die „Unterdrückung“ des Hohenpriesters erwähnt wird (s. o.), dieses Ereignis zeitlich zu fixieren. In 2,10ff. wird ein Priester $N s-J m n$ anstelle seines (inzwischen verstorbenen) Vaters vernommen. Er gibt zu, daß sein Vater an dem Verbrechen (von einem Tragschrein o. ä. wird Kupfer gestohlen) beteiligt war, aber er selbst war damals noch ein Kind ( $(\underline{d} d \stackrel{s}{s} \mathrm{r}$ ). Bei einer erneuten Befragung benennt er einen ‘ $\not 3 w t j-n f r$, den er zu der betreffenden Stelle gehen sah. Dieser ' ḩswtj-nfr macht die oben zitierte Aussage (pMayer A, 6,4ff.), daß er im 6. Monat der „Unterdrückung“ des Hohenpriesters verschleppt worden ist, und als er zurückkam, war der Tragschrein beraubt und verbrannt. Peet schließt daraus, daß das Verbrechen eben in der Abwesenheit des 'b̧ $3 w t j-n f r$ geschah, und da zu dieser Zeit $N s-J m n$ ein Kind war, jetzt aber schon Priester ist, müsse es mindestens 3-4 Jahre her $\operatorname{sein}^{62}$. Diese Folgerung ist jedoch irrig: Es steht überhaupt nichts davon im Text, daß das Verbrechen in dieser Zeit geschah! Der Aussage des 'ḅswtj-nfr läßt sich nur eines entnehmen: Der Schrein ist nach seiner Rückkehr schon zerstört (und der Brand ist zweifellos bei der Erstürmung oder Besetzung Medinet Habus passiert: Bei der Vernehmung bezüglich des Verbrechens ist immer nur von Diebstahl die Rede). Der Zeuge möchte sich nicht, wie Peet wohl vermutet hat, mit dieser Aussage ein Alibi verschaffen, sondern er will sagen, daß er erst

\footnotetext{
${ }^{57}$ Für diesen angeblichen Machthunger des Hohenpriesters wird gern auf die Darstellung bei seiner „Belohnungsinschrift“ in Karnak (Lefebvre, Inscriptions concernant les Grands Prêtres, pl. II) verwiesen. Piotrovski hat schon 1929 zu Recht darauf verwiesen, daß es sich bei dem dort abgebildeten König um eine Statue handelt, s. die Verweise bei Federn, CdE 34, $1959,214$.

${ }^{58}$ MIO 4, 1956, 174ff.; Geschichte des alten Ägypten, p. 203.

${ }^{59} \mathrm{CAH}^{2}$, p. 633.

${ }^{60}$ Fs Fairman, p. 94.

${ }^{61}$ S. Černý, Community, p. 108.

${ }^{62}$ JEA 12, 1926, 256-7; in JEA 14, 1928, 67 geht er sogar von 4-5 Jahren aus. Kees, Herihor, p. 5: „Im Mindestfall 4-5, im Höchstfalle 10-15 Jahre“.
} 
seit dieser Zeit mit dem betreffenden Gegenstand bzw. dem Ort, wo er aufbewahrt wird, etwas zu schaffen hat, wie aus seiner folgenden Aussage deutlich hervorgeht (und was vom Gericht offenbar akzeptiert wird, denn dieser Zeuge wird nicht „peinlich befragt“). Es läßt sich der Aussage nur entnehmen, daß das Verbrechen irgendwann vorher geschehen ist.

Abgesehen davon wird die Aussage des Ns-Jmn, er sei noch ein kleines Kind gewesen, so ernst nicht zu nehmen sein, wie Peet selbst zugibt ${ }^{63}$, denn er hat natürlich jedes Interesse daran, zur Zeit des Diebstahls so klein und unschuldig wie möglich gewesen zu sein. Die Richter scheinen ihm dies auch nicht abzunehmen, da er trotz dieser Aussage erneut vernommen wird.

- Das gleiche gilt für die Aussage des Webers $W n$-nbt in pMayer A, 2,17ff., der wieder für seinen verstorbenen Vater vernommen wird. Auch aus dieser Aussage geht nichts Genaues über den Zeitpunkt des Verbrechens hervor. Er sagt allerdings, daß sein Vater getötet ( $\underline{b} d b)$ wurde, als er ein Kind $\left(\breve{s} r j^{64}\right)$ war, und es ist sehr wahrscheinlich (wenn auch nicht sicher), daß dieser Tod mit den kriegerischen Ereignissen zusammenhängt. Auch hier liegt es natürlich wieder im Interesse des Zeugen, sein damaliges Alter möglichst gering anzugegeben. War er zum Zeitpunkt der Vernehmung 14/15 und beim Tod des Vaters 12/13, ergäbe sich keinerlei Problem für den Ansatz des Krieges ab Jahr 17.

- In seiner wenige Jahre später erschienenen Abhandlung ${ }^{65}$ hat Kees diese Schlußfolgerung Peets nicht übernommen, vermutlich weil er ihre Unfundiertheit bemerkt hatte. Das Argument, das Kees nun für einen Ansatz des „Krieges“ etliche Jahre vor Jahr 19 bringt (p. 5), ist auch nicht besser: In der zitierten Zeugenaussage des Arbeiters (kswtj) 'b̧wtj-nfr heißt es, damals, als die Barbaren den Tempel stürmten und er verschleppt wurde, habe er Esel seines Vaters gehütet. Kees schließt daraus, er könne also damals höchstens 12 Jahre alt gewesen sein. Das ist ganz aus der Luft gegriffen: Warum sollte er nicht mit 13, 15 oder 18 Esel gehütet haben? Vielleicht hatte der Vater ja gar keinen anderen Sohn. Überdies kann er, wenn er von Soldaten verschleppt wurde, so klein nicht gewesen sein, da sie ihn ja sicher als eine Art „Leibburschen“ o. ä. mitgenommen haben werden.

Den Aussagen der Grabräuberpapyri läßt sich nicht entnehmen, wie lange die kriegerischen Ereignisse zurückliegen. Allzu lange kann es nicht sein, da die Erinnerung der Zeugen noch sehr lebhaft und detailliert ist, aber einen genauen Ansatz erlauben diese Texte nicht.

Ich hoffe, es ist deutlich geworden, daß die Argumente dafür, die in den Grabräuberpapyri und in der Karnak-Inschrift des Hohenpriesters Amenhotep erwähnten Auseinandersetzungen lägen längere Zeit vor der whm-mswt-Zeit, alle nicht stichhaltig sind. Das heißt aber auch, es besteht nicht mehr der geringste Grund, an der seit Kees ${ }^{66}$ gängigen Theorie festzuhalten, Panehsi sei dem Hohenpriester Amenhotep zu Hilfe gekommen. Wenn er wirklich der Retter im Auftrag des Königs war, wieso ist er dann wenig später plötzlich ein Feind? Umgekehrt gibt es aber sehr gute Gründe, die Ereignisse zwischen Jahr 17 und Jahr 19 zu legen: Erstens eben die inzwischen erfolgte Verfemung des Panehsi, die alle bisherigen Theorien nicht erklären können ${ }^{67}$. Zweitens sagt im pBM 10383, II,5 ein Wächter des „Hauses des Pharao“ (wohl der Palast in Medinet $\mathrm{Habu}^{68}$ ), daß er seinen Posten verließ, als Panehsi kam und seinen Vorgesetzten ( $p 3 j . j$ brj) attackierte $(t h j)$. Mit diesem Vorgesetzten kann doch nur der Hohepriester gemeint $\operatorname{sein}^{69}$, zumal derselbe Terminus thj auch im Zusammenhang mit der „Unterdrückung“ des Amenhotep erscheint. Diese Aussage kann man durchaus als positiven Beweis dafür ansehen, daß es Panehsi war, der den Krieg gegen den Hohepriester führt, und da dieser seinerseits den König gegen seinen Feind zu Hilfe ruft, ist es klar, warum Panehsi später in offiziellen Dokumenten verfemt ist.

Mit dieser m. E. viel wahrscheinlicheren Annahme, der Vizekönig Panehsi sei Verursacher der „Unter-

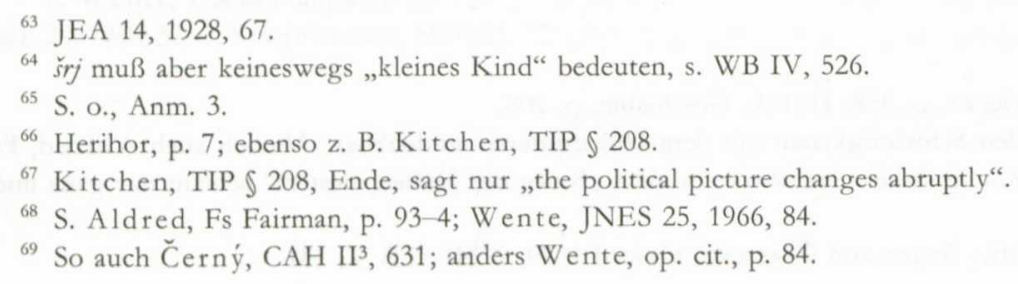


drückung" des Hohenpriesters, läßt sich eher ein zusammenhängendes Bild der Ereignisse gewinnen. Man kann sich den Ablauf vielleicht folgendermaßen vorstellen:

1. In den späteren Jahren Ramses' IX. und unter Ramses X. scheinen die Zustände in der Thebais äußerst unsicher zu sein: Man hört von umherschweifenden „Wüstenbewohnern“ und Libyern; die Furcht vor ihnen läßt die Nekropolenarbeiter nicht zur Arbeit gehen ${ }^{70}$. In derselben Zeit findet die erste Serie der Grabräuberprozesse statt, die gleichfalls darauf schließen lassen, daß die Administration nicht mehr in der Lage war, die innere Sicherheit aufrechtzuerhalten. Die permanente ökonomische Krise am Ende der 20. Dynastie wird die Lage noch explosiver gemacht haben.

2. Es ist deshalb gut möglich, daß Ramses XI. (am ehesten zu Beginn seiner Herrschaft) den Vizekönig Panehsi anwies, mit nubischen Truppen zu intervenieren, um mit Waffengewalt für Ordnung zu sorgen. Die Anwesenheit des Panehsi in Theben könnte sich leicht von daher erklären.

3. Da die wirtschaftliche Lage schlecht war, dürfte Panehsi nach einiger Zeit vor dem Hauptproblem gestanden haben, das alle Heerführer bis in die Neuzeit fast überwiegend beschäftigt hat: Der Unterhalt der Truppen.

4. Diese Lage dürfte dazu geführt haben, daß Panehsi entweder vom König zum Speichervorsteher ernannt wurde, um seine Truppen zu versorgen, oder um generell eine gerechte Bewirtschaftung notfalls gewaltsam durchsetzen zu können, oder daß er sich vielleicht sogar eigenmächtig dieses Amt anmaßte. Die erwähnte Verringerung der Zahl der Nekropolenarbeiter im Jahr 8 Ramses' XI. könnte ebenfalls auf Versorgungsschwierigkeiten zurückgehen, die durch die Anwesenheit von Truppen ja noch verschärft werden mußte.

5. Es ist wohl eher unwahrscheinlich, daß Panehsi von vornherein einen coup d'état geplant hatte. Vermutlich ergaben sich aus seiner Stellung als Speichervorsteher und seinem Bemühen, seine Truppen zu versorgen, automatisch Kompetenzstreitigkeiten mit dem Hohenpriester des Amun als dem Chef der Amundomäne, der größten Wirtschaftsinstitution in Oberägypten. Man vergleiche z. B. die „Belohnungsinschrift" eben dieses Hohenpriesters Amenhotep unter Ramses IX.71 , wo der Hohepriester ja gerade für seinen Eifer beim Eintreiben und Abführen von Abgaben belobigt wird. Man kann sich leicht vorstellen, daß die (bezeugten) Aktivitäten des Panehsi in dieser Richtung (vgl. RAD, p. 36 ff.) zu Konflikten führten, und diese Konflikte wird Panehsi schließlich durch militärisches Vorgehen gegen den Hohenpriester zu lösen versucht haben.

6. Nach dem Angriff des Panehsi ist Amenhotep, wie seine Inschrift berichtet, den König um Hilfe angegangen, und aus der Intervention des Königs wird sich der Krieg entwickelt haben, der dann auch im Norden geführt wurde und (u. a.) zur Zerstörung von Kynopolis führte. Es ist mit Nachdruck darauf hinzuweisen, daß keine Rede davon sein kann, daß sich der Krieg nur nördlich bis Kynopolis (und nicht darüber hinaus) erstreckte, wie einige Autoren anzunehmen scheinen ${ }^{72}$. Die Erwähnung von Kynopolis in pBM 10052, 10,18 erfolgt vielmehr aus rein persönlichen Gründen des betreffenden Zeugen, ist also bloßer Zufall. In pMayer A, 13, B2 wird deutlich gesagt, daß auch in Unterägypten (' mbtj) Krieg geführt wurde. Offenbar ist Panehsi mit Nachdruck gegen die königliche Macht vorgegangen und bis Unterägypten vorgedrungen ${ }^{73}$.

7. Man kann an dieser Stelle eine, zugegeben ziemlich spekulative, Erwägung einschieben: Bekanntlich wird zu Beginn der 21. Dynastie die nördliche Hauptstadt von Tell ed-Dab a - Qantir (also der alten Ramsesstadt) nach Tanis verlegt (erste signifikante Erwähnung im Wenamun), und dies muß relativ kurzfristig geschehen sein, da schon bald darauf die Ramsesstadt nicht mehr in den Quellen erscheint. M. Bietak hat als Grund dafür die Versandung des pelusischen Nilarms und seinen partiellen Abfluß in den tanitischen Nilarm bei Bubastis angegeben ${ }^{74}$, und das ist auch überzeugend. Man fragt sich aber,

\footnotetext{
${ }^{70}$ S. Černý, p. cit., 616-8.

${ }^{71}$ Helck, MIO 4, 1956, $161 \mathrm{ff}$.

72 Z. B. Grimal, Histoire de l'Egypte ancienne, p. 358; Helck, Geschichte, p. 203.

${ }^{73}$ Der Krieg in 'mbtj wäre bei bloß lokalen Schwierigkeiten mit dem Hohenpriester undenkbar. Ähnlich auch Aldred, Fs Fairman, p. 95-6, aber seine ursächliche Trennung dieser Operation von dem „Krieg des Hohenpriesters“ scheint mir ganz und gar unwahrscheinlich.

${ }^{74}$ Tell ed-Dab a, II, Wien 1975, 99 ff.; 215 ff.; Avaris and Piramesse, Oxford 1979, 273 ff.; LÄ V, 131.
} 
ob das allein der Grund gewesen sein kann; in der 21./22. Dynastie existierte ja auch ein Kanal zwischen beiden Orten ${ }^{75}$, so daß u. U. Tanis auch ohne Verlagerung der gesamten Stadt hätte als Hafen genutzt werden können. Außerdem scheint die Verlagerung ziemlich plötzlich erfolgt zu sein. Wäre es nicht möglich, daß die Ramsesstadt im Verlauf von Panehsis Kämpfen in Unterägypten zerstört wurde? In einem solchen Fall wäre es nur natürlich, daß man dann den Wiederaufbau gleich an einer besser geeigneten Stelle begann. Vielleicht läßt sich ja die Frage eines Tages archäologisch entscheiden (Brandspuren etc.). 8. Nach den Kämpfen in Unterägypten müssen königliche Truppen Panehsi zurückgeschlagen und schließlich bis nach Nubien zurückgedrängt haben, wo die Late Ramesside Letters bekanntlich bis zum Jahr 10 (wḥm mswt) Kämpfe belegen. Diese königlichen Truppen werden unter dem Befehl des Pianch gestanden haben, der dann konsequenterweise alle Titel und Funktionen des Panehsi übernimmt.

9. Wie Aldred ${ }^{76}$ überzeugend dargelegt hat, können die ausgedehnten Plünderungen der Königsgräber in Theben-West kaum von kleineren Räuberbanden durchgeführt worden sein, sondern sie werden das Werk der (sich zurückziehenden) nubischen Truppen des Panehsi gewesen sein, möglicherweise mit dessen ausdrücklicher Billigung zwecks Auffüllung der Kriegskasse, wie Aldred meint.

10. Unmittelbar nach dem Krieg, in dem vermutlich ausgedehnte Zerstörungen angerichtet und eben die Königsgräber und sicher noch manches andere geplündert und verwüstet wurden, wird man die Ära $w h \mathfrak{m} m$ swt eingeführt haben, wahrscheinlich, um ein Zeichen für den Wiederaufbau des Landes zu setzen.

11. Pianch übernimmt dann zu den Ämtern und Funktionen des Panehsi auch noch die Würde des Hohenpriesters $^{77}$ (wohl nach dem Tod des Amenhotep, der ja schon sehr lange im Amt war) sowie die Funktion des Wezirs ${ }^{78}$. Offenbar hat Pianch eben dadurch (mit Billigung des Königs) das erreicht, was Panehsi (gegen den König) nicht geschafft hatte.

12. Nach dem Tod des Pianch übernimmt sein Schwiegersohn Herihor dessen Ämter, nach dem vermutlich nicht allzu lange danach erfolgten Tod des Königs ${ }^{79}$ dann sogar (für bestimmte Bereiche) den Königstitel.

Über die Herkunft des Herihor ist, wie über die des Pianch, nichts bekannt. Es ist nicht unwahrscheinlich, daß auch er aus Offizierskreisen stammt. Es ist schon oft bemerkt worden, daß einige seiner Kinder libysche Namen $\operatorname{tragen}^{80}$. Es wäre also gut möglich, daß Herihor libyscher Herkunft war. Da ja bekanntermaßen libysche Söldner am Ende des Neuen Reichs eine sehr wichtige Rolle spielen, könnte man spekulieren, ob nicht Pianch versuchte, diese Leute an sich zu binden, indem er einen ihrer Führer zu seinem Schwiegersohn machte. Aber derlei läßt sich natürlich nicht belegen.

${ }^{75}$ S. Bietak, Tell ed-Dab a, II, 214.

${ }^{76}$ Fs Fairman, p. 96-8.

77 Ob Pianch schon während der zweiten Welle von Grabräuberprozessen, also in den Jahren 1-2 der whm mswt, Hoherpriester war, stehe dahin. Die Nichterwähnung des Hohenpriesters ließe sich vielleicht so erklären, weil er ja zu der Zeit Krieg in Nubien führte.

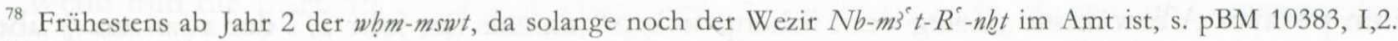

79 Während der Grabräuberprozesse im Jahr 1-2 der whm mswt, also in seinem 19. und 20. Jahr, scheint Ramses XI. politisch noch aktiv zu sein, vgl. Aldred; op. cit., p. 95 oben. Über sein weiteres Schicksal ist nichts bekannt, man hat praktisch nur die Jahreszahlen. Ein Passus aus dem Brief pBerlin 10487 des Pianch an T3ry (s. LRL, 36,11-12, etwa aus dem Jahr 10 der $w h m m s w t)$ : kjtj mdt: jr pr-3 j.jrj.f pḥ p̧j ts mj jb mj jb; br jr pr-3 hrj n-m m-r' „Another matter: As for Pharaoh, 1.p.h., how shall he reach this land? And of whom is Pharaoh, l.p.h., superior still?" ist verschiedentlich so verstanden worden, daß Pianch hier mehr oder weniger verächtlich vom König spricht (z. B. Wente, JNES 25, 1966, 85). Das muß nicht unbedingt sein. Man könnte es auch so verstehen: Der König hat irgendwie seine Absicht erkennen lassen, dem Pianch in Nubien zu Hilfe zu kommen. Pianchs Antwort könnte einfach bedeuten: Welche Transportkapazitäten und welche Truppen hat er denn (als rhetorische Frage: Er hat eben keine und kann deshalb auch nicht kommen).

${ }^{80}$ S. Wente, Übersetzungsheft zu ToK, I, p. XIII-XIV; Leahy, Libyan Studies 16, 1985, 55; TIP $\int 211$; von Beckerath, RdE 20, 1968, 32-3; Stadelmann, MDAIK 27, 1971, 120, n. 45; LÄ II, 1129; Kitchen, in: Libya and Egypt (London 1990), p. 23. 


\section{Exkurs 1: Zum Hohenpriester „Ramsesnacht II.“}

Bierbrier hat sich vor einiger Zeit ${ }^{81}$ dafür ausgesprochen, nach dem Hohenpriester Amenhotep am Ende der 20. Dynastie einen weiteren Hohenpriester namens Ramsesnacht einzuschieben, der spätestens im 8. Jahr Ramses' X. im Amt gewesen wäre ${ }^{82}$. Träfe das zu, wäre natürlich die oben gegebene Rekonstruktion der Ereignisse völlig unmöglich, da dann der „Krieg des Hohenpriesters“ Amenhotep nichts mit Panehsi zu tun haben könnte.

Bierbrier geht aus von dem thebanischen Graffito 1860a, in dem im Jahr 8 eines ungenannten Königs ein Hoherpriester Ramsesnacht, ein königlicher Mundschenk $R^{c}$-ḅr-wnm.f, ein Bürgermeister Amenmose und ein Vorarbeiter ( $3 n j z t$ ) Amunnacht erwähnt werden. Dafür, in diesen Personen nicht den bis zum Jahr 2 Ramses' IX. belegten bekannten Hohenpriester Ramsesnacht und den zuletzt im Jahr 3 Ramses’ IV. belegten Bürgermeister Amenmose zu erblicken, führt Bierbrier folgende Gründe an:

1. Ein ' $3 n j z t$ Jmn-nbt ist erst für das Jahr 3 Ramses' X. bezeugt. Vorher sei kein Vorarbeiter dieses Namens zu belegen und keiner einschiebbar. Dieses Argument ist von Bell in aller Ausführlichkeit widerlegt worden ${ }^{83}$, so daß ich darauf nicht weiter einzugehen brauche.

2. Der Bürgermeister Amenmose könne hier nicht zur Datierung herangezogen werden, da er aufgrund der genealogischen Daten der Familien des $T 3-n f r$ und des B3k-n-Hnzw so alt sein müßte, daß er kaum das 8. Jahr Ramses ' VI. erreicht haben könnte (zumal nicht im Amt). Dieses Argument ist schon von Bell $^{84}$ aus eher generellen Gründen bezweifelt worden. Speziell läßt sich noch folgendes einwenden: Die Altersberechnungen Bierbriers ${ }^{85}$ gehen zurück auf Seeles ${ }^{86}$ unglückliche Rekonstruktion dieser Familien. Nach ihm (p. 7) müßten 4 Generationen (davon zwei weibliche Glieder!) 165 Jahre umspannt haben, fast eine Unmöglichkeit: Man müßte dann eine Serie von Spätgeburten ansetzen. Die ganze Sache hängt nur an der Verknüpfung der Familie des T3-nfr mit der des (Hohenpriesters) B3k-n-Hn₹w. Auf pl. 17 bei Seele (op. cit.) wird ein Amunpriester (kein Hoherpriester!) B3k-n-Hnzw mit einer Frau Mr.s-gr als $j t j n$ mwt $n$ bmt.f $(. f=\underline{T} 3-n f r)$ bezeichnet. Es bieten sich hier zwei Möglichkeiten an: Entweder es handelt sich trotz der Namensgleichheit der Frau gar nicht um den bekannten Hohenpriester (immerhin wird er ja nicht so bezeichnet) oder $j t j$ ist hier, wie auch sonst manchmal, als Großvater bzw. Vorfahr zu verstehen: Dann könnte man statt der 4 Generationen leicht 5-7 verrechnen. Läßt man diese unglückliche Verknüpfung beiseite, ergibt sich ein ganz klares Bild:

Der Sohn des $\underline{T} 3-n f r$, der 3. Amunprophet $J m n-m-j p t\left(, \Pi I^{“}\right)$ ist der Schwiegersohn des Hohenpriesters Ramsesnacht („,I“) $)^{87}$. Also müßte sein Onkel, der Bruder seiner Mutter Nfrt-jrj, nämlich eben der im Graffito genannte Bürgermeister Amenmose, zur selben Generation wie der Hohepriester Ramsesnacht („'“') gehören, der, wie erwähnt, mindestens bis ins 2. Jahr Ramses’ IX. amtierte. Ein Erscheinen des Amenmose im 8. Jahr Ramses' VI. hätte mithin überhaupt nichts Erstaunliches.

3. Wenn nach Bierbrier ${ }^{88}$ schon im 8. Jahr Ramses' X. ein Hoherpriester Ramsesnacht („II“) im Amt war, hieße das, daß der Hohepriester Amenhotep schon vorher gestorben bzw. nicht mehr im Amt war, mithin der im pMayer A erwähnte „Krieg des Hohenpriesters“ damals (in Jahr $1 / 2$ der whm-mswt) über 20 Jahre zurücklag! Auch Bierbrier hat diese Schwierigkeit bemerkt ${ }^{89}$. Ich halte das für ganz ausgeschlossen: Das Verbrechen, das im pMayer A (Sektion I, II, IV) verhandelt wird, liegt noch vor diesem Krieg (s. o., p. 28-29) und die Zeugen haben noch ein ganz erstaunlich detailliertes Erinnerungsvermögen: Sie wissen genau, was sie damals durchs „Schlüsselloch“ gesehen haben, was gestohlen wurde, wieviel Getreide sie exakt dafür gaben. In pMayer A, 6.3ff. sagt der Zeuge 'b̧swtj-nfr, daß ein bestimmter Gegen-

${ }^{81}$ JEA 58, 1972, 195-199.

82 Vgl. auch JEA 61, 1975, 251.

${ }^{83}$ Serapis 6, 1980, 7-16.

${ }^{84}$ Op. cit., p. 15 , n. 98.

85 JEA 58, 1972, 196-7; The Late New Kingdom in Egypt, Warminster 1975, p. 5-6.

${ }^{86}$ The Tomb of Tjanefer at Thebes, OIP 86, 1959, p. 5-7.

${ }^{87}$ S. Bierbrier, Late New Kingdom, p. 6/9.

88 JEA 61, 1975, 251.

${ }^{89}$ JEA $61,1975,251:$, $\ldots$ this fact certainly complicates the problem of the suppression of the high priest Amenhotep.“ 
stand bei der Besetzung Medinet Habus während der „Unterdrückung“ des Hohenpriesters Amenhotep verbrannte (vermutlich beim Sturm auf die Festung). Die Holzreste davon werden nach Wiederherstellung der Ordnung von einigen Beamten eingesammelt und versiegelt, und 'b̧ $w$ w $j$ - $n f r$ sagt, daß das Siegel darauf intakt „bis heute“, also zur Zeit der Aussage, ist! Das wäre doch kaum möglich, wenn das Ereignis Jahrzehnte zurückläge!

Man kann aus alldem nur folgern, daß es keinen Hohenpriester Ramsesnacht II gegeben hat.

\section{Exkurs 2: Die Datierung der Hohenpriesterinschrift aus Karnak}

Kürzlich hat Helck versucht ${ }^{90}$, die von Wente in JNES 25, 1966, 73ff. neuveröffentlichte Inschrift, in der ein im erhaltenen Fragment ungenannt bleibender Hoherpriester berichtet, von einem Feind 8 Monate lang „unterdrückt“ (thj) worden zu sein, dem Hohenpriester Ramsesnacht zuzuweisen, nicht seinem Nachfolger Amenhotep, wie Wente es getan hatte. Er argumentiert dabei folgendermaßen:

1. Die Namensbeischrift der Priesterfigur (Z. 30/31) liest er rückläufig (Z. 31-30), wobei der Rest von Z. 30 an das (ergänzte) untere Ende von Z. 31 anschlösse, so daß der erhaltene Teil der Beischrift nur Titel und Namen des Mrj-B3stt, des Vaters des Hohenpriesters Ramsesnacht wiedergäbe. Die obere Hälfte von Z. 31 müßte dann Titel und Namen des Ramsesnacht enthalten, so daß der Platz für eine weitere Generation, also Amenhotep, sehr knapp würde, diese Möglichkeit aber, wie Helck zugibt (p. 54), auch nach seiner Rekonstruktion nicht auszuschließen ist.

Davon abgesehen ist seine Rekonstruktion und Lesefolge Z. 31-30 überhaupt ganz unsicher:

- Über der Hand in Z. 30 kann noch viel gestanden haben (nur das unterste Drittel des Textes ist erhalten), so daß eine Folge Z. 30-31 ohne weiteres möglich wäre ${ }^{91}$.

- Es könnte rechts neben Z. 31 in der oben fehlenden Partie eine Z. 32 gegeben haben.

- Es ist unsicher, ob der hier genannte Mrj-B3stt wirklich Mrj-B3stt „I“, der Vater des Ramsesnacht, ist. Möglich wäre auch ein Bruder ${ }^{92}$ (oder ein Sohn) des Amenhotep als derjenige, der diesen Text für Amenhotep gravieren ließ.

Man kann also nur den Schluß ziehen, daß die Beischrift, so wie sie erhalten ist, über die Identität des angegriffenen Hohenpriesters überhaupt nichts aussagt.

2. In Z. 3 wird der Totentempel Ramses' VI. erwähnt. Weil dieser Totentempel nur hier sicher bezeugt ist, folgert Helck, daß er nie fertiggestellt und wirtschaftlich nicht funktionsfähig war, man also nur in der Regierungszeit Ramses' VI. ein Amt darin hätte haben können. Dies ist für Helck sogar das entscheidende Argument dafür, den Text in die Zeit Ramses' VI. zu datieren ${ }^{93}$. Es ist aber zugleich ein klassisches argumentum e silentio: Bei unserer bruchstückhaften Kenntnis dieser Zeit ist es absolut unerheblich, ob der Tempel einmal oder fünfmal erwähnt wird. Daraus läßt sich gar nichts folgern.

3. „Wenn nun die Inschrift in Zeile 4 weiter davon spricht, daß, , [S. M. mir die Gunst wiederholte und er mich einsetzte als Oberdomänenvorsteher des Totentempels] Ramses' III. im Amuntempel auf der Westseite von Theben', so paßt das vorzüglich zu der Tatsache, daß Ramsesnacht dieses Amt nach der bereits zitierten Inschrift im Grab TT 148 verwaltet hat. Von seinem Sohn, dem Hohenpriester Amenophis, ist diese Tatsache nicht bekannt! ‘94

Die Inschrift spricht eben nicht davon, sondern Helck spricht in seiner Ergänzung davon. Es ist nur in irgendeinem Zusammenhang vom [Totentempel] Ramses' III. die Rede.

4. In Z. 6 wird gesagt, daß der Vater des redenden Hohenpriesters im Jahr [X] des pr- 3 [...] im Westen von Theben beerdigt wurde. Nun verweist $p r-{ }^{~} 3$ in dieser Zeit normalerweise auf den regierenden König, aber es gibt auch Ausnahmen, s. Wente, op. cit., p. 84, n. 34, so daß Wentes Ergänzung pr-'3

\footnotetext{
${ }^{90}$ OrNS 53, 1984, 52-6.

${ }^{2}$ S. a. KRI VI, 538,11.

${ }^{92}$ So Kitchen, TIP \207, n. 21; vgl. Bierbrier, op. cit., p. 11-2.

${ }^{93}$ Op. cit., p. 56.

94 Op. cit., p. 54

Z. ägypt. Sprache u. Altert.kd. 119 (1992) 1
} 
[Ramses IX.] durchaus möglich ist ${ }^{95}$. Wente zieht auch eine weitere Möglichkeit in Betracht ${ }^{96}$ : Ramsesnacht könnte zugunsten seines Sohnes zurückgetreten und dann tatsächlich erst unter Ramses XI. gestorben sein. Beide Möglichkeiten sind von Helck nicht widerlegt worden.

5. Helcks Behauptung (p. 55), der Text stamme aus dem 5. Jahr Ramses' VI., beruht nur auf seiner falschen Übersetzung von Z. 16 (richtig bei Wente).

Von Helcks Argumenten ist kein einziges überzeugend. Dagegen wäre es extrem unwahrscheinlich, wenn innerhalb einer relativ kurzen Zeit zwei Hohepriester „attackiert“ worden wären (zumal die Ägypter solche Dinge ja ohnehin nur höchst ungern erwähnen!), und das dann auch noch für eine fast gleich lange Zeit ${ }^{97}$.

\section{Zur Chronologie der 21. Dynastie}

Es ist oben schon kurz angesprochen worden, daß die im Zusammenhang mit Herihor überlieferten Jahreszahlen 5 und 6 wohl besser als seine eigenen „Regierungsjahre“ zu verstehen sind. Das bedarf sicher einer eingehenderen Begründung.

Es gibt seit längerer Zeit in der Ägyptologie eine Art Dogma, daß die thebanischen Hohenpriester der 21. Dynastie keine eigenen Regierungsjahre gezählt haben, ein Dogma, weil es nur selten begründet wird ${ }^{98}$. Folgende Gründe lassen sich dafür anführen:

1. Es gebe keine ausdrücklich auf den Hohenpriester bezogenen Daten (z. B. mit $n$ oder $b r)^{99}$.

2. Es gibt aber - in der 2. Hälfte der 21. Dynastie - ausdrücklich auf den König bezogene Daten: TIP \ 379 ff., Nr. 54; 56; 73-4; 77; 82-84; z. T. mit zusätzlicher Nennung des Hohenpriesters (Nr. 73; vgl. auch 53), wodurch klar ist, daß nach dem König, nicht dem Hohenpriester datiert wurde.

3. Da diese Belege aber ausschließlich aus Zeiten stammen, wo der entsprechende Hohepriester keine königlichen Titel und Attribute beanspruchte, könnte man denken, daß es bei diesen anders sei. Dagegen wenden die Gegner von Hohenpriester-Datierungen wiederum folgendes ein ${ }^{100}$ :

Selbst Pajnedjem I., der „königlichste“ von ihnen, zähle keine eigenen Regierungsjahre: Es gibt eine Serie von Daten für Jahr 1-15 (z. T.) mit Pajnedjem als Hohenpriester (TIP \381-2). Im Jahr 16 aber erscheint sein Sohn Masaharta als Hoherpriester und Pajnedjem als König (ibid., §383, 27). Folgerung: Pajnedjem habe sich zwischen diesen Daten den Königstitel zugelegt, aber keine neue Zählung begonnen, denn sonst müßte es ja Jahr 1 statt Jahr 16 heißen. Außerdem ist der König Pajnedjem noch in einem Jahr 8 bezeugt, das demnach auf einen Regierungswechsel in Tanis, nicht in Theben, hinweisen sollte.

Es gibt aber durchaus auch einige Fakten, die auf eine andere Lösung deuten.

1. Man hat eine Art komplementärer Distribution:

Diejenigen Hohenpriester, die (mitunter) königliche Titel führen, sind Herihor, Pajnedjem und Mencheperre. Während ihrer Amtszeit ist in Theben bzw. Oberägypten kein einziges offizielles Dokument der tanitischen Könige bezeugt, ganz anders als in der zweiten Hälfte der Dynastie:

- Von Smendes ist in Karnak (sehr wahrscheinlich jedenfalls) ein Graffito belegt ${ }^{101}$, also kein offizielles Dokument. Die Felsstele bei Dababieh ${ }^{102}$ (südlich von Theben) könnte man natürlich so verstehen, aber das ist keineswegs notwendig. Es ist ohnehin unstrittig, daß zwischen den tanitischen Königen und den thebanischen Hohenpriestern kein prinzipieller politischer Gegensatz bestand, sondern eher freundschaftliche und familiäre Verbindungen; warum sollte also Smendes keine Hilfe bei einer Restauration schicken,

\footnotetext{
${ }^{95}$ Ein Beleg für $p r-3+$ Kartusche aus der 21. Dynastie in Rec. Trav. 22, 1900, p. 53 unten.

${ }^{9}$ Op. cit., p. 83; auf Vorschlag von Baer.

97 Vgl. auch Bierbrier, op. cit., p. 13.

${ }_{98}$ S. Mapero, Momies Royales, p. $723 \mathrm{f}$; Y Young, JARCE 2, 1963, 101-4; TIP $₫ 23$, n. 112; $₫ 62$. Vgl. auch die rigorose Haltung von Kees, Hohenpriester, p. 52 oben.

99 D. h. es gibt eines, das aber ignoriert oder uminterpretiert wird, s. zuletzt TIP2 $₫$ 435; 501 .

100 S. TIP $\int 23$, n. $112 ; \int 62$.

101 S. TIP $\{214$, n. 65.

102 S. RecTrav 10, 1888, 135-6.
} 
wenn er dazu in der Lage war? Das muß noch lange nicht bedeuten, daß auch nach ihm datiert wurde. Die Felsstele scheint denn auch (dem Textaufbau nach, wenn auch mehr als die Hälfte fehlt) kein Datum zu enthalten, obwohl man das bei einer derartigen Inschrift unbedingt erwarten sollte!

- Von Jmn-m-njswt ist in Oberägypten nichts belegt, was angesichts seiner wahrscheinlich sehr kurzen Regierungszeit ohnehin nicht verwunderlich ist.

- Ebensowenig ist aber von Psusennes bezeugt, und das ist schon erheblich wichtiger: Bei einer solch langen Regierungszeit, wie man sie ihm zuschreibt, sollte man doch unbedingt einige Belege erwarten. - Mit Amenemope ändert sich das Bild abrupt. Von ihm an sind alle tanitischen Könige gut in Theben bezeugt ${ }^{103}$, obgleich sie alle erheblich kürzer regiert haben als z. B. Smendes oder Psusennes. Zugleich beanspruchen die zu ihrer Zeit amtierenden Hohenpriester von Theben keinerlei königliche Würde mehr. Das gilt zumindest für Pajnedjem II. Das haarige Problem Psusennes II./III. soll hier (als vorläufig unlösbar) ausgeklammert bleiben.

Man kann also - als Alternative zum bisherigen Datierungssystem - folgende Möglichkeit ins Auge fassen:

In der ersten Hälfte der 21. Dynastie wird nach den Hohenpriestern in Theben, die königliche Würden beanspruchen, datiert, das sind Herihor, Pajnedjem I. und Mencheperre ${ }^{104}$, nach dem Tode Mencheperres, dessen Endzeit ungefähr mit dem Regierungsbeginn Amenemopes zusammenfällt (s. u.), aber nach den Königen in Tanis (wobei, wie gesagt, der Fall Psusennes II./III. unsicher ist und hier nicht weiter betrachtet wird).

Der berühmte Stoffetzen mit einem Jahr $49^{105}$ ist zweifellos mit Kitchen ${ }^{106}$ als Doppeldatierung zu verstehen. Ich würde es aber vorziehen, [Jahr X des] König Amenemope; Jahr 49 [des Hohenpriesters Mencheperre] zu ergänzen. Für eine Koregenz Psusennes I./Amenemope gibt es, wie überhaupt für Koregenzen in der 21. Dynastie, keinerlei handfeste Anhaltspunkte ${ }^{107}$, wohl aber für die Gleichzeitigkeit des Endes der Amtszeit des Mencheperre mit dem Beginn der Regierung des Amenemope und ebenso für Regierungsjahre des Mencheperre, vgl. TIP $\ 388,51=$ ASAE 8, 1907, 30 (105): Auf derselben Mumie ein Band aus dem 48. Jahr des(!) Hohenpriesters Mencheperre und ein Band aus einem Jahr 1, 4. $3 \mathrm{bt}, 1$, das (mit Kitchen) nur dem Amenemope zugerechnet werden kann. Auf diese Weise ist es unnötig, bei der Doppeldatierung eine Koregenz zu postulieren.

Diese Alternative hat folgende Vorteile:

Die oben ausgeführte Komplementarität - während der Amtszeit der „königlichen“ Hohenpriester keinerlei offizielle Dokumente und keine Daten mit den Namen der gleichzeitigen tanitischen Könige, unter dem „nichtköniglichen“ Pajnedjem II. dagegen eine ganze Reihe von Bezeugungen und Daten dieser Könige - würde sich so erklären, ebenso das Jahr 48 des Mencheperre. Daß außer diesem einen keine weiteren ausdrücklich auf den Hohenpriester bezogenen Daten vorliegen, dürfte sich damit erklären, daß die Hohenpriester, auch wenn sie Regierungsjahre zählten, nie als wirkliche Könige betrachtet worden sind (oder sich selbst so gesehen haben). Auch dieses eine Datum dürfte einen besonderen Grund haben (s. u.).

Akzeptiert man diese Alternative, müßte man alle Daten, in denen Pajnedjem I. (als König oder Hoherpriester) vorkommt ${ }^{108}$, auf dessen Amtszeit beziehen ${ }^{109}$. Der einzige Einwand dagegen wäre, daß Pajnedjem I. in seinem Jahr 8 als König erscheint, im Jahr 12 und 13 dagegen wieder als Hoherpriester ${ }^{110}$. Das wäre aber nur dann verwunderlich, falls es wirklich eine zeitliche Entwicklung gab: Pajnedjem I.

\footnotetext{
${ }^{103}$ S. TIP \& $388 \mathrm{ff}$.

104 Zu Masaharta s. u.

105 TIP $\int 387,47$.

${ }^{106}$ TIP $₫ 29$.

${ }^{107}$ Vgl. auch Wente, JNES 26, 1967, 155, n. 4; Murnane, Ancient Egyptian Coregencies (SAOC 40, 1977), p. $186-7 ; 235$.

${ }^{108}$ S. TIP \ $381-4$; § $386,40(-41)$.

${ }^{109}$ Das oben (Anm. 47) erwähnte Band mit einem Jahr 1 des Pajnedjem auf der Mumie der Nedjemet muß natürlich keineswegs bedeuten, daß sie in diesem Jahr 1 starb! Man vergleiche die Mumie Pajnedjems II., auf der Bänder aus den Jahren 1, 3, 7, 9 gefunden wurden, die Kitchen (TIP \389) mit gutem Grund Siamun zuwies; gestorben ist Pajnedjem II. bekanntlich im Jahr 10.

${ }^{110}$ S. TIP $\int 382,22 / 25 ; 386,40(-41)$.
} 
war zunächst Hoherpriester, dann übernahm sein Sohn Masaharta das Amt und Pajnedjem wurde König. Das ist aber schon deshalb nicht zwingend, weil Pajnedjem auch da, wo er klar Hoherpriester ist, deutlich königliche Attribute annimmt ${ }^{111}$. Auch bei dem Hohenpriester Mencheperre ${ }^{112}$ ist keine Entwicklung vom Hohenpriester zum König zu erkennen: Sein Name wird keineswegs selten in der Kartusche geschrieben ${ }^{113}$, aber noch in den Belegen aus dem Jahr 48, also aus dem Ende seiner Amtszeit, wird er nur als Hoherpriester erwähnt. Welche Kriterien bei der Wahl königlicher Attribute eine Rolle spielten, wissen wir nicht, aber auf jeden Fall kann man das Führen des Königstitels nicht dazu nutzen, die Belege zeitlich zu ordnen.

Youngs Behauptung ${ }^{114}$, das Zeugnis für den König Pajnedjem I. aus einem Jahr 8 sei aufgrund der Handschrift mit den Zeugnissen für den Hohenpriester Mencheperre aus den Jahren 6 und 7 zusammenzustellen, ist ganz unfundiert, s. Wente, JNES 26, 169, n.115.

Der Übergang von Jahr 15 zu Jahr 16 Pajnedjems I. bedeutet also nur, daß er damals das Hohepriesteramt seinem Sohn Masaharta übergeben hat und daher nach Jahr 15 nur noch als König erscheinen könnte, nicht mehr als Hoherpriester, nicht aber, daß er nicht schon vorher „König“ war.

Konsequenterweise müssen auch die beiden ersten Daten auf der "Stele der Verbannten“ (Z. 1; 4: jeweils Jahr 25) auf Pajnedjem bezogen werden. Das in Z. 4 entspricht offenbar dem Zeitpunkt, wo Amun (nach dem Tode des Masaharta) den Mencheperre als Hohenpriester einsetzt. In Z. 8 ist dann mit Sicherheit eine niedrigere Jahreszahl zu ergänzen ${ }^{115}$, die also in den Anfang der Amtszeit des Mencheperre fallen sollte. Das darauf geschilderte Orakel des Amun betreffs der Rückrufung von Verbannten wird eine Art Amnestie ${ }^{116}$ sein, wie sie ja für den Beginn neuer Regentschaften typisch ist. Zwischen diesen Daten, dem Jahr 25 und dem Jahr X des Mencheperre (vermutlich sehr kurz nach Jahr 25) muß der Tod des Pajnedjem liegen und damit der Beginn einer neuen Jahreszählung durch Mencheperre. Von den vier ersten Hohenpriestern der 21. Dynastie hätte mithin nur Masaharta keine eigenen Regierungsjahre gezählt, weil seine Amtszeit vollständig in die Regierungszeit des Pajnedjem I. fiel.

Ein Problem bleibt jetzt noch der Hohepriester $N s$ - $b 3-n b-\underline{D} d t$. Er ist einmal in Theben als Hoherpriester belegt ${ }^{117}$ und er ist, wie Pajnedjem II., ein Sohn Mencheperres. Da Pajnedjem II. noch unter Siamun bezeugt ist, muß $N s-b 3-n b-D d t$ vor ihm amtiert haben, also der direkte Nachfolger des Mencheperre sein. Außerdem ist ein Hoherpriester $N s-b 3-n b-\underline{D} d t$ auf Armbändern im Grab des Psusennes in Tanis bezeugt ${ }^{118}$. Wenn der thebanische und der tanitische $N s-b 3-n b-\underline{D} d t$ dieselbe Person sind, wäre aber die obige Annahme scheinbar nicht möglich, die Datierungen in die Jahre 48 und 49 als Jahreszählung des Hohenpriesters Mencheperre zu verstehen, denn wenn Mencheperre noch im ersten und zweiten Jahr des Amenemope Hoherpriester war, hätte $N s-b s-n b-\underline{D} d t$ beim Begräbnis des Psusennes nicht als solcher erscheinen dürfen, falls es sich um dieselbe Person handelt.

Einen Ausweg aus diesem Dilemma hat Niwiński ${ }^{119}$ gefunden: Es kann sich durchaus um dieselbe Person handeln, aber zur Zeit des Begräbnisses des Psusennes ist $N s$ - $b 3-n b-D d t$ Hoherpriester des Amun in Tanis und offenbar erst später (für sicher nur sehr kurze Zeit) in Theben. Wenn auch die Konflikte, die Niwiński andeutet ${ }^{120}$, nicht nachzuweisen sind, dürfte es in dieser Zeit doch zu entscheidenden Ereig-

111 S. Wente, JNES 26, 1967, 163, n. 59 sowie Kees, Hohenpriester, p. 24. Vgl. v. a. die Statue Kairo CG 42191, die Inschriften auf den Sockeln der Sphingen am 1. Pylon (s. Barguet, Le Temple d'Amon-Rê, p. 42), wo der Hohepriester P. sagt „Es gibt keinen König, der getan hat, was ich getan habe“, die Inschrift auf der Basis des kleinen Tempels von Medinet Habu (LD, Text, III, 164), in der der Hohepriester P. nțr nfr genannt und mit einer ganzen Serie königlicher Epitheta versehen wird, das Graffito aus dem Grab Ramses' XI. (s. Cicarello, in: Brooklyn Museum, Theban Expedition, 1908, zitiert bei Römer [s. o., Anm. 13], p. 581) mit dem Hohepriestertitel als Thronnamen und in der 2. Kartusche Pajnedjem $m 3^{\circ}-b r w$ !

112 Vgl. TIP \& 501.

${ }^{113}$ Außer den Belegen bei Kitchen, loc. cit., vgl. auch ASAE 8, 1907, 22, Nr. 11. Hier ist zudem bemerkenswert, daß auf ein- und derselben Mumie sein Name einmal mit, einmal ohne Kartusche geschrieben wird.

${ }^{114}$ JARCE 2, 1963, $102 . \quad{ }^{115}$ S. v. Beckerath, RdE 20, 1968, 33, mit n. 2.

${ }_{116}$ Des Mencheperre, der ja auch den Orakelvorgang durch seine Frage an Amun in Gang setzt.

117 ASAE 8, 1907, 35, Nr. 135.

118 TIP S $387,48$.

119 JARCE 16, 1979, 59-60; Coffins, \43.

${ }^{120}$ Coffins, p. 51 oben. 
nissen gekommen sein. Mit dem Regierungsantritt Amenemopes ist ja - nach der hier vorgeschlagenen Deutung - ein Wechsel im System der Datierungen verbunden, was sicher gravierende Gründe gehabt haben wird. Man kann vermuten, daß die Tatsache, daß im Jahr 48 des Mencheperre, also wohl im 1. Jahr des Amenemope ${ }^{121}$, zum ersten Mal ein Datum ausdrücklich auf einen Hohenpriester bezogen wird, mit diesem Wechsel zusammenhängt: Es war eben zum ersten Mal nötig, klar zu sagen, auf wen sich das Datum bezog.

Welche Konsequenz hätte ein solches Datierungssystem nun für die absolute Chronologie? Zunächst hätte man nur für die tanitischen Könige ab Amenemope Daten aus zeitgenössischen Quellen, dürfte also bis dahin nur die Hohenpriesterdatierungen verrechnen; man kann die manethonischen Daten für Smendes, Nefercheres und Psusennes also ignorieren. Die Addition sieht folgendermaßen aus: Für Psusennes II./III. sind 13 Jahre belegt, es wäre also nur billig, die 14 Jahre bei Manetho (Africanus) anzusetzen. Für Siamun sind 17 Jahre belegt, für Osochor 2 (aber auch hier wäre die manethonische Zahl, 6 Jahre, wohl vorzuziehen) und für Amenemope $10^{122}$. Für den Hohenpriester Mencheperre sind wohl 47 Jahre zu verrechnen ${ }^{123}$, für Pajnedjem kaum mehr als 25 Jahre (Stele der Verbannten, s. o.). Bei Herihor ist als höchstes ein Jahr 6 belegt. Wegen der oben erwähnten Schwierigkeit aber, daß im Jahr 5 eine Expedition losgeschickt wird, Holz für die Barke des Amun zu holen, auf diese Expedition (als gelungen) aber in der Dekoration des Chonstempels angespielt wird, sollte man Herihor mindestens 10 Jahre zugestehen. Es ergibt sich also $14+17+6+10+47+25+10=129$. Das kommt der in allen Manetho-Versionen einhellig belegten Summenzahl von 130 Jahren für die 21. Dynastie verblüffend nahe. Wenn man nun als Gegenprobe die manethonischen Zahlen für Smendes, Psusennes und Nefercheres (die parallel zu den Hohenpriestern herrschten) zu den Daten der Könige ab Amenemope rechnet, ergeben sich (je nach Version) 118 bzw. 123 Jahre. Das muß nicht unbedingt ein Widerspruch sein, denn es ist keineswegs sicher, ob Smendes gleichzeitig mit Herihor begonnen hat, Regierungsjahre zu zählen. War er, wie einige meinen, der Sohn des Herihor, wäre es sogar wahrscheinlich, daß seine eigene Zählung erst nach Herihor einsetzte.

121 S. Niwiński, Coffins, p. 48 (2).

122 Nicht sicher, s. TIP \388, 55: Falls der Beleg zu Siamun gehört, könnte man zumindest Manethos 9 Jahre übernehmen.

${ }^{123}$ Falls Jahr 1 des Amenemope = Jahr 48 des Mencheperre ist, s. o., Anm. 121. 